\title{
Metastasis suppressor 1 controls osteoblast differentiation and bone homeostasis through regulating Src-Wnt/ $\beta$-catenin signaling
}

\section{Meng Chen}

Tianjin Medical University Chu Hsien-I Meomorial Hospital

\section{Liying Shan}

Tianjin Medical University Chu Hsien-I Memorial Hospital

\section{Ying Gan}

Tianjin Medical University Chu Hsien-I Memorial Hospital

\section{Lijie Tian}

Tianjin Medical University Chu Hsien-I Memorial Hospital

\section{Jie Zhou}

Tianjin Medical University Chu Momorial Hospital

\section{Endong Zhu}

Tianjin Medical University Chu Memorial Hospital

\section{Hairui Yuan}

Tianjin Medical University Chu Hsien-I Memorial Hospital

\section{Xiaoxia Li}

Tianjin Medical University

\section{Baoli Wang ( $\nabla$ blwang@tmu.edu.cn )}

Tianjin Medical University Insitite of Endocrinology https://orcid.org/0000-0002-0470-1651

\section{Research Article}

Keywords: osteoblast, differentiation, Metastasis suppressor 1, Src, Wnt/ $\beta$-catenin

Posted Date: November 1st, 2021

DOl: https://doi.org/10.21203/rs.3.rs-976455/v1

License: (c) (i) This work is licensed under a Creative Commons Attribution 4.0 International License. Read Full License January 30th, 2022. See the published version at https://doi.org/10.1007/s00018-022-04147-y. 

Metastasis suppressor 1 controls osteoblast differentiation and bone homeostasis through regulating Src-Wnt/ $\beta$-catenin signaling Meng Chen ${ }^{1 \S}$, Liying Shan ${ }^{1 \S}$, Ying Gan ${ }^{1}$, Lijie Tian $^{1}$, Jie Zhou ${ }^{1}$, Endong Zhu' ${ }^{1}$, Hairui Yuan ${ }^{1}$, Xiaoxia Li $^{2}$, Baoli Wang ${ }^{1 *}$

${ }^{1} \mathrm{NHC}$ Key Lab of Hormones and Development, Tianjin Key Lab of Metabolic Diseases, Chu

6 Hsien-I Memorial Hospital \& Institute of Endocrinology, Tianjin Medical University, Tianjin

7 300134, China

$8 \quad{ }^{2}$ College of Basic Medical Sciences, Tianjin Medical University, Tianjin 300070, China

$9 \quad$ Running Title: MTSS1 promotes osteogenesis

10 *Correspondence to: Baoli Wang, E-mail: blwang@tmu.edu.cn

11 Address: 6 Huan-Rui-Bei Road

Tianjin Medical University Chu Hsien-I Memorial Hospital \& Institute of Endocrinology

Tianjin 300134, China 
Abstract

Metastasis suppressor 1 (MTSS1) plays an inhibitory role in tumorigenesis and metastasis of a variety of cancers. To date, the function of MTSS1 in the differentiation of marrow stromal progenitor cells is completely unknown. In the current study, we explored whether and how MTSS1 has a role in osteoblast differentiation and bone homeostasis. Our data showed that MTSS1 mRNA was upregulated during osteoblast differentiation and downregulated in the osteoblastic lineage cells of ovariectomized and aged mice. Functional studies revealed that MTSS1 promoted the osteogenic differentiation from marrow stromal progenitor cells. Mechanistic explorations uncovered that the inactivation of Src and afterwards activation of canonical Wnt signaling were involved in osteoblast differentiation induced by MTSS1. The enhanced osteogenic differentiation induced by MTSS1 overexpression was attenuated when Src was simultaneously overexpressed, and conversely, the inhibition of osteogenic differentiation by MTSS1 siRNA was rescued when the Src inhibitor was supplemented to the culture. Finally, the in vivo transfection of MTSS1 siRNA to the marrow of mice significantly reduced the trabecular bone mass, along with the reduction of trabecular osteoblasts, the accumulation of marrow adipocytes, and the increase of phospho-Src positive cells on the trabeculae. No change in the number of osteoclasts was observed. This study has for the first time unraveled that MTSS1 contributes to osteoblast differentiation and bone homeostasis through regulating Src-Wnt/ $\beta$-catenin signaling. It also suggests the potential of MTSS1 as a new target for the treatment of osteoporosis.

Key words: osteoblast; differentiation; Metastasis suppressor $1, \mathrm{Src}$; Wnt/ $\beta$-catenin 


\section{Abbreviations}

37 ALP: alkaline phosphatase

$38 \quad$ BMP2: bone morphogenetic protein 2

39 BMSCs: Bone marrow mesenchymal stem cells

40 C/EBP: CCAAT/enhancer binding protein

41 FBS: fetal bovine serum

42 GSK3 $\beta$ : glycogen synthase kinase $3 \beta$

43 HRP: horseradish peroxidase

44 LRP6: low-density lipoprotein receptor-related protein 6

45 MTSS1: Metastasis suppressor 1

46 OPN: osteopontin

47 PPARY: peroxisome proliferator-activated receptor $\mathrm{Y}$

48 Quantitative reverse transcription polymerase chain reaction: qRT-PCR

49 Runx2: runt-related transcription factor 2

50 siRNA: small interfering RNA

51 TCF7L2: transcription factor 7 like 2

52 TRAP: tartrate-resistant acid phosphatase 


\section{Introduction}

54 Bone marrow mesenchymal stem cells (BMSCs) have the potential to differentiate toward

55 multiple cell types including osteoblasts, adipocytes, chondrocytes and myoblasts. Under 56 normal physiological conditions, the capabilities of BMSCs to differentiate toward osteoblastic and adipogenic lineages are similar and therefore a reciprocal and competitive relationship usually exists between osteogenesis and adipogenesis [1, 2]. Certain pathological conditions might render BMSCs more committed toward one aspect of osteogenesis or adipogenesis at 60 the expense of the other aspect, thereby lead to metabolic bone disorders such as osteoporosis and osteopetrosis, etc [1].

The modulation of osteogenic and adipogenic commitment of BMSCs is finely orchestrated by a variety of transcription factors and signaling pathways including runt related transcription factor-2 (Runx2), osterix, bone morphogenetic proteins (BMPs), and Wnt/ß-catenin pathway, which usually positively controls osteogenic differentiation [3-7]. In contrast, the transcription factors, i.e., peroxisome proliferator activated receptor $\mathrm{Y}$ (PPARY) and CCAAT/enhancer binding proteins (C/EBPs) are the major positive regulators of adipocyte differentiation [8, 9].

Metastasis suppressor 1 (MTSS1), also referred to as missing in metastasis (MIM), is a cytoskeletal scaffold protein which binds to actin and affects cytoskeleton organization [10]. MTSS1 is widely expressed in various tissues, e.g., spleen, thymus, testis, prostate, uterus, colon and peripheral blood [11]. Accumulating evidences have established the inhibitory function of MTSS1 in tumorigenesis and metastasis of a variety of cancers including the carcinomas of bladder [12, 13], stomach [14], breast [15], pancreas [16], colorectum [17], and 
lung [18]. Its low expression in these cancers is associated with poor prognosis. However, in the past few years, controversial findings arose which identified MTSS1 to be a metastasis driver in several types of tumors such as melanoma [19] and hepatitis B-related hepatocellular carcinoma [20].

To date, the function of MTSS1 in the differentiation of marrow stromal progenitor cells is completely unknown. In the current study, we explored whether and how MTSS1 has a role in osteoblast differentiation and bone homeostasis. The data uncovered the pro-osteogenic function of MTSS1 in vitro and in vivo and elucidated the mechanism involved in the processes. skeletal muscle, brown and white fat (Fig. 1A). The expression profiling of MTSS1 during 


\section{MTSS1 stimulated osteogenic differentiation of stromal progenitor cells}

To elucidate the role of MTSS1 in osteogenesis, loss-of-function and gain-of-function experiments were performed. For the loss-of-function study, qRT-PCR and Western blotting analysis verified the efficacy of the MTSS1 shRNA lentivirus in silencing the endogenous expression of MTSS1 in primary BMSCs (Fig. 2A). Functionally, after osteogenic treatment, the knockdown of MTSS1 in primary BMSCs suppressed osteogenic differentiation, which was evidenced by the attenuation of alkaline phosphatase (ALP) staining and alizarin red staining (Fig. 2B). Accordingly, the mRNA and/or protein expression levels of the osteogenic factors such as Runx2, osterix, ALP and osteopontin were reduced in the cells underexpressing MTSS1 as compared to those infected with control virus (mRNAs reduced by $30 \sim 87 \%$ and proteins reduced by $60 \sim 88 \%$ ) (Fig. 2C, D). Moreover, MTSS1 silencing in ST2 cells following the transfection of MTSS1 small interfering RNAs (siRNAs) also attenuated the osteogenic differentiation as compared to the control cells (Fg. 2E-H).

For the gain-of-function study, qRT-PCR analysis verified the overexpression of MTSS1 in ST2 following the transfection of the MTSS1 expression construct (Fig. 3A). Functionally, after osteogenic treatment, the overexpression of MTSS1 in ST2 cells stimulated osteogenic differentiation, which was evidenced by the enhancement of ALP staining (Fig. 3B). Accordingly, the mRNA and/or protein expression levels of the osteogenic factors were increased in the cells overexpressing MTSS1 as compared to those transfected with the vector (mRNAs increased by 1.55 3.29-fold and proteins increased by 42 68\%) (Fig. 3C, D). Furthermore, MTSS1 overexpression did not significantly change the cell growth rate of ST2 cells (Fig. 3E). 

cells

We then tried to uncover the mechanism by which MTSS1 regulates osteoblast differentiation. The data showed that the dysregulation of MTSS1 altered Src signaling. Briefly, Western blotting analysis revealed that MTSS1 overexpression inhibited, while MTSS1 depletion stimulated the phosphorylation of Src (Y419) in ST2 cells (Fig. 4A, B). The results from Co-IP experiment revealed that Src1 physically interacted with MTSS1 and low-density lipoprotein receptor-related protein 6 (LRP6) (Fig. 4C). Moreover, Western blotting analysis showed that the overexpression of MTSS1 significantly increased, while its knockdown decreased the protein levels of phosphorylated LRP6 (S1490), phosphorylated glycogen synthase kinase $3 \beta$ (GSK3 $\beta$ ) (S9), non-phospho- $\beta$-catenin and transcription factor 7 like 2 (TCF7L2) (Fig. 4D, E). Consistently, the overexpression of Src significantly decreased phosphorylated GSK3 $\beta$ (S9), non-phospho- $\beta$-catenin and TCF7L2 (Fig. 4F).

Dysregulation of Src expression impaired osteogenic differentiation of progenitor cells gain-of-function experiment was performed. qRT-PCR and Western blotting analysis verified suppressed osteogenic differentiation, as evidenced by the attenuation of ALP staining (Fig. 5B). Accordingly, the mRNA and/or protein levels of the osteogenic factors were reduced in the cells overexpressing Src as compared to those transfected with vector (mRNAs reduced 
by $46 \sim 56 \%$ and proteins reduced by 50 61\%) (Fig. 5C, D). Furthermore, Src mRNA was elevated in the BMSCs of ovariectomized mice (Fig. 5E).

Moreover, we performed cotransfection experiment to investigate if $\mathrm{Src}$ is able to relieve the disturbed osteogenesis induced by MTSS1. The results showed that under osteogenic condition, the cells cotransfected with MTSS1 expression construct and vector displayed enhanced ALP staining and increased levels of osteogenic factors as compared to the cells transfected with vector only. This stimulatory effect of MTSS1 on osteogenesis was attenuated when the cells were cotransfected with MTSS1 and Src expression constructs (Fig. 6A-C). Conversely, the cells receiving MTSS1 siRNA and vehicle displayed repressed ALP staining and decreased levels of osteogenic factors as compared to the cells receiving control siRNA and vehicle. This anti-osteogenic effect of MTSS1 siRNA was attenuated when the cells received MTSS1 siRNA and and Src inhibitor 1 (Src-11) (Fig. 6D-E).

\section{Silencing of MTSS1 impaired the differentiation of osteoblasts and reduced trabecular}

\section{bone mass in mice}

We further investigated the physiological role of MTSS1 in the differentiation of BMSCs in mice. qRT-PCR analysis demonstrated that the in vivo transfection of MTSS1 siRNA is effective in knocking down the endogenous expression of MTSS1 in the BMSCs of the tibiae (Fig. 7A, B). Histomorphometric analysis using $\mu \mathrm{CT}$ showed that 4 weeks after the in vivo transfection, bone volume per tissue volume (BV/TV), bone mineral content per tissue volume (BMC/TV) and trabecular thickness (Tb.Th) were significantly decreased by $25 \%, 23 \%$ and 17\%, respectively, in the mice receiving MTSS1 siRNA vs. those receiving control siRNA (Fig. 7C-F). By contrast, the trabecular number (Tb.N) was not changed in the transfected 
mice (Fig. 7G).

We quantified the number of adipocytes, osteoblasts and osteoclasts on the sections H\&E staining analysis showed that both the number and area of the adipocytes were increased in the mice receiving MTSS1 siRNA as compared to those receiving control siRNA (Fig. 8A-C). Immunohistochemical staining analysis showed that the ALP-positive osteoblasts were reduced in the mice receiving MTSS1 siRNA vs. those receiving control siRNA (Fig. 8D, E). Moreover, the immunohistochemical staining analysis also showed that the mice receiving MTSS1 siRNA had more phospho-Src-positive cells on the trabeculae than the control mice (Fig. 8F, G). By contrast, tartrate-resistant acid phosphatase (TRAP) staining analysis did not reveal any change in the number of osteoclasts (Fig. 8H, I). Furthermore, qRT-PCR analysis revealed that the overexpression of MTSS1 in ST2 cells did not change the ratio of RANKL to OPG (Fig. 8J).

\section{Discussion}

Previously, the research interests in MTSS1 were mainly focused on its function in tumorigenesis and metastasis of various cancers. In this study we aimed to find some novel function of MTSS1 in bone. Based on the observation that MTSS1 was upregulated during osteoblast differentiation and downregulated in the osteoblastic lineage cells from the ovariectomized mice and aged mice, we hypothesized that MTSS1 may have a regulatory role in osteogenesis and bone homeostasis.

To delineate the exact role of MTSS1 in osteogenesis, loss-of-function and gain-of-function experiments were applied using primary BMSCs and/or stromal cell line. It appeared that MTSS1 did not affect the cell growth rate of the progenitor cells. However, it 
was shown that the overexpression of MTSS1 stimulated, while the depletion of MTSS1 inhibited the differentiation of osteoblasts and the expression of the osteogenic transcription factors and marker genes. Of more importance, in vivo experiments showed that the depletion of MTSS1 in the marrow resulted in the reduction of trabecular osteoblasts and the accumulation of marrow fat. Additionally, the trabecular bone mass was reduced in the mice following the knockdown of MTSS1 in the marrow, as indicated by the decline of trabecular bone volume and bone mineral content. Of interest, it appears that the reduction of bone mass was mainly due to the reduction in the thickness of the trabeculae, while the number of trabeculae was not affected. Based on the observation that osteoclast number was similar between the two groups of mice, we concluded that the reduction of trabecular bone mass is a result of the impaired formation of mature osteoblasts.

MTSS1 is one of the defining members of the I-BAR family of negative membrane curvature-sensing proteins that was initially identified to be absent in metastatic bladder cancer [11]. It contains a C-terminal WH2 actin-binding motif and functions as a cytoskeletal remodeling protein [10]. MTSS1 is now considered to be the antagonist of Src family of tyrosine kinases (SFK) in a variety of cellular activities. Quinones et al. provided the first genetic evidence that MTSS1 modulated directed cell migration by suppressing endocytosis and the activities of the CD2-associated protein/cortactin complex [21]. Another group reported that the MTSS1-dependent antagonism of cortactin in promoting ciliogenesis was mediated by the SFK [22]. Consistently, MTSS1 loss in mice resulted in increased SFK activity in Purkinje neurons, degenerating Purkinje neurons with low firing rates, and cell death [23]. In our study, we demonstrated that MTSS1 directly interacted with Src. Of interest, 
we also observed the hypo-phosphorylation of Src following the overexpression of MTSS1 and hyper-phosphorylation of Src after the knockdown of MTSS1 in stromal progenitor cells. These data suggest that Src acts in response to MTSS1 to mediate the regulatory function of 210 the latter in stromal progenitor cells.

The proto-oncogene Src has long been studied about its role in bone homeostasis.

212 Based on the evidences from genetic animal models, Src is detrimental to bone mass accrual

213 because Src null mice displayed severe osteopetrosis [24]. The cellular basis for the 214 phenotype involved the impairment in both osteoclasts and osteoblasts. Src is critical for the normal cytoskeletal architecture of osteoclasts [25]. Src null mice had more osteoclasts which did not form ruffled borders, thus functioned inappropriately and failed to resorb bone [26].

217 Moreover, the deletion of Src enhanced osteoblast differentiation and bone formation, contributing to the increase of bone mass as well [27]. In our study, we investigated the role of Src in vitro. It was shown that Src was able to suppress the differentiation of osteoblasts. Additionally, overexpression of Src in the progenitor cells attenuated the stimulation of osteoblast differentiation induced by MTSS1, and inhibition of Src activity in the progenitor cells attenuated the blockade of osteoblast differentiation induced by MTSS1 siRNA. These evidences further support the interaction of MTSS1 with Src in regulating osteogenic commitment. Of more interest, the mice receiving MTSS1 siRNA exhibited more phospho-Src in the trabecular bone cells, indicating the involvement of Src in the reduction of bone mass observed in the mice transfected with MTSS1 siRNA. 
that Src interacts with canonical Wnt signaling, exhibiting either stimulatory or inhibitory effect

230 on the latter depending on cellular context. In cancer cells, Src contributes to tumor 231 progression at least partially through enhancing canonical Wnt signaling [28, 29]. Src acted as a positive regulator of $\beta$-catenin, which functioned by docking to and being activated by Dishevelled-2 in response to Wnt stimulation [30]. Activated Src downregulated E-cadherin or phosphorylated $\beta$-catenin at $\operatorname{Tyr}(654)$, as a result facilitating the release of $\beta$-catenin from cadherin complex at the membrane and promoting accumulation of $\beta$-catenin in the nucleus, thus rendering it available for transcriptional regulation [31, 32]. Of interest, Chen et al. recently reported that in non-cancer cells such as mouse embryonic fibroblasts (MEF), Src was both sufficient and necessary for suppressing canonical Wnt pathway [33]. Src associated with LRP6, directly phosphorylated LRP6 at several conserved tyrosine residues, leading to the removal of LRP6 from cell surface and the disruption of LRP6 signalosome formation, which eventually blocked the signal transduction of canonical Wnt signaling [33]. This appears to account for the fact that Src and canonical Wnt signaling have opposite effects on osteoblastogenesis and bone formation.

In this study, the Co-IP experiment demonstrated the physical binding of Src to LRP6, suggesting that Src might directly phosphorylate LRP6 in osteoblastic cell lineage. 246 Furthermore, MTSS1 increased, while Src decreased the level of phospho-GSK3 $\beta$ (S9), 247 non-phospho- $\beta$-catenin and TCF7L2. Collectively, our data provide evidences that, consistent 248 to Chen et al's report, Src in osteoblastic lineage also suppressed the activation of

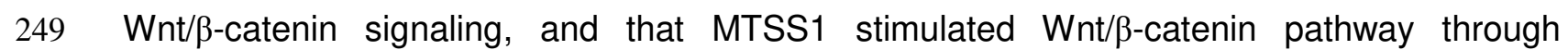
antagonizing the non-receptor tyrosine kinase Src. 


\section{Conclusion}

252

In summary, we have identified a new osteogenic factor, MTSS1, which positively

253 regulates the osteogenic differentiation of marrow stromal progenitor cells in vitro and in vivo,

254 and contributes to the homeostasis of bone. Mechanistic investigations have revealed that

255 the inactivation of Src and activation of $\mathrm{Wnt} / \beta$-catenin signaling mediates the function of

256 MTSS1 in the cell fate determination of bone marrow stromal progenitor cells and the

257 homeostasis of bone (Fig. 9). Taken as a whole, the current study suggests that MTSS1 is a

258 novel target for the therapeutic intervention of metabolic bone disorders such as

259 osteoporosis.

260 Materials and methods

261 Cells

262 ST2 cells were maintained in a-MEM supplemented with $10 \%$ fetal bovine serum (FBS).

263 Primary murine BMSCs were collected from the femurs and tibiae of mice. Briefly, the bone

264 marrow was flushed from 6-week-old C57BL/6 mice with a-MEM using a 26-gauge syringe

265 and the marrow cells were seeded in a $10 \mathrm{~cm}$ dish at the density of $3 \times 10^{5} / \mathrm{cm}^{2}$ in $\alpha-M E M$

266 supplemented with $20 \%$ FBS. After culturing for 3 days, the non-adherent hematopoietic cells

267 were removed by changing the culture medium with $\alpha$-MEM containing $10 \%$ FBS. The

268 medium was refreshed every 3 days. At $70-80 \%$ confluence, the cells were trypsinized by

$2690.25 \%$ trypsin/EDTA and passaged. The cells at passage 3-5 were induced to allow

270 differentiation using osteogenic medium containing $50 \mu \mathrm{g} / \mathrm{mL}$ ascorbic acid and $10 \mathrm{mM}$

$271 \beta$-glycerophosphate. After 3 days of induction, the cells underwent RNA and protein extraction and after 14 days or 21 days of induction, the cells underwent ALP staining or 
alizarin red staining to evaluate the status of differentiation.

\section{Cell proliferation assay.}

275 The cells were grown in a 96-well plate, transfected with vector or the construct overexpressing MTSS1 at $60-70 \%$ cell confluence using JetPRIME transfection reagent

277 (Polyplus, Illkirch, France) following the supplier's instructions. The effect of MTSS1 was assessed by using a CCK-8 cell counting kit (Vazyme, Nanjing, China).

\section{siRNAs, construct and transfections}

For the gain-of-function studies, the gene sequence coding for full-length Src were PCR-amplified, respectively, then cloned into the eukaryotic expression vector pcDNA3.1(+) at the sites of BamHI/EcoRI using the ClonExpress II One Step Cloning Kit (Vazyme, Nanjing, China). The expression construct of MTSS1 was purchased from Origene (Rockville, MD, USA). Each construct or the vector was transfected into ST2 cells at the confluence of $70-80 \%$ using JetPRIME transfection reagent. After 4 hrs of transfection, the transfection medium was confluence to allow osteogenic differentiation.

For the loss-of-function studies, the siRNAs specifically targeting the coding sequence of MTSS1 or Src were designed and synthesized (Genepharma, Shanghai, China). Each target or control siRNA, at the concentration of $20 \mathrm{nmol} / \mathrm{L}$, was transfected into ST2 cells at $50-60 \%$ confluence using lipofectamine RNAi-max reagent (Invitrogen, Carlsbad, CA, USA). After 24 hrs of transfection, the transfection medium was replaced with complete culture medium. The cells were induced at appropriate confluence to allow osteogenic differentiation. 
respectively.

\section{qRT-PCR}

Total RNA was extracted from tissues using RNAiso Plus (Takara, Dalian, China), or from cells using a RNA extraction kit (Omega, USA) following the supplier's instructions. $0.5 \mu \mathrm{g}$ RNA was reverse transcribed using the MMLV reverse transcriptase (Thermo Fisher, Waltham, MA, USA) in presence of oligo(dT) and random primer. The resulting cDNAs were used as templates for quantitative PCR using the SYBR-Green Master PCR Mix (Abclonal, Wuhan, China). The relative expression level of each target gene was calculated following the comparative $\Delta \Delta \mathrm{Ct}$ method with $\beta$-actin as the internal control for normalization. The primers for the PCR reactions are listed in Supplemental Table 1.

\section{Western blot analysis}

Cells were harvested in RIPA lysis buffer and protein contents of the cell lysates were quantified by a BCA Assay kit (Beyotime, Shanghai, China). Proteins were separated on $10-15 \%$ sodium dodecyl sulfate-polyacrylamide gel electrophoresis (SDS-PAGE) and transferred onto nitrocellulose membranes. After blocking with 5\% skim milk, the membranes were probed with primary antibodies at $4{ }^{\circ} \mathrm{C}$ overnight, then incubated with horseradish peroxidase (HRP)-conjugated secondary antibodies. The immunoreactive bands were detected using a chemiluminescence reagent (Proteintech, Wuhan, China). $\beta$-actin was used as a loading control and the relative protein expression level of each target gene was calculated by dividing the optical density of the target gene with that of $\beta$-actin. The primary antibodies used are as follows: antibodies from Abcam (Cambridge, MA, USA): anti-TCF7L2 (ab76151), anti-LRP6 (ab134146), anti-osterix (ab94744), and anti-ALP (ab108337); 
antibodies from Cell Signaling Technology (Danvers, MA, USA): anti-Runx2 (\#12556), anti-non-phospho- $\beta$-catenin (\#8814), anti-phospho-LRP6 (S1490) (\#2568), anti-GSK3 $\beta$

319 (\#12456), and anti-phospho-GSK3ß (S9) (\#5558); antibodies from Abclonal (Wuhan, China): anti-MTSS1 (A11697), and anti-Src (A19119); antibody from Affinity (Changzhou, China): anti-Phospho-Src (Y419) (AF3162); antibodies from Proteintech (Wuhan, China): anti- $\beta$-catenin (51067-2-AP), anti-osteopontin (25715-1-AP), and anti- $\beta$-actin (66009-1-lg).

\section{Lentiviral packaging and infection}

To carry out loss-of-function experiment in primary BMSCs, a pair of oilgos coding for the shRNA of MTSS1 were annealed, then ligated to pLVX-shRNA2 vector at BamHI/EcoRI sites (Clontech, Mountain View, CA, USA). The lentiviruses were packaged using a kit from Jiman Biotech (Shanghai, China) following the supplier's instructions. Briefly, the lentiviral shRNA expression construct or the vector was transfected, respectively, into 293T cells together with the packaging plasmids. $48 \mathrm{~h}$ after transfection, the supernatant containing the viruses was collected, purified, and titered. The primary BMSCs were infected with the virus with a multiplicity of infection (MOI) of 20 , then induced with osteogenic medium at $70-80 \%$ confluence to allow differentiation.

\section{Alkaline phosphatase (ALP) staining and alizarin red staining}

After 14 days of osteogenic treatment, the differentiated osteoblasts underwent ALP staining.

Briefly, the cell cultures were rinsed with PBS, fixed for 10 min with $4 \%$ paraformaldehyde, then stained for 20 min with NBT/BCIP staining solution (Beyotime, Shanghai, China). The differentiated osteoblasts underwent alizarin red staining after 21 days of osteogenic 
for 15 min with $1 \%$ alizarin red stain solution $(\mathrm{pH} 4.2)$.

\section{Co-immunoprecipitation (Co-IP)}

341 The cells were washed with ice-cold phosphate buffered saline (PBS) and lysed with RIPA

342 buffer supplemented with protease inhibitors. After lysis, $500 \mu \mathrm{g}$ of each protein sample was

343 incubated at $4{ }^{\circ} \mathrm{C}$ with the primary antibody against Src or rabbit IgG. To capture the

344 immunocomplexes, protein $\mathrm{A} / \mathrm{G}$ magnetic beads were added and incubated for $2 \mathrm{hrs}$ at $4^{\circ} \mathrm{C}$.

345 The supernatant was discarded and the beads were washed with PBS plus $0.5 \%$ Triton X100,

346 and then boiled in SDS sample buffer. The denatured samples were then subjected to

347 SDS-PAGE and immunoblot analysis for MTSS1 and LRP6.

\section{In vivo delivery of siRNAs}

349 C57BL/6 mice were obtained from SPF Biotechnology (Beijing, China) and housed in SPF facility. The mice had free access to food and water and were kept with a controlled $12 \mathrm{~h}$ light : $12 \mathrm{~h}$ dark cycle. To deliver the siRNAs to the bone marrow of mice, 2'-Ome modified MTSS1 siRNA or control siRNA (Genepharma, Shanghai, China) was formulated with in vivo-jetPEI (Polyplus, Illkirch, France) following the supplier's instructions. Briefly, for one mouse, $11 \mu \mathrm{g}$ siRNA and $1.5 \mu \mathrm{L}$ in vivo-jetPEI were diluted, respectively, with $12.5 \mu \mathrm{l}$ of $5 \%$ glucose and then mixed. Afterwards, the formulated mixture was injected to the marrow cavity of the complete $\alpha$-MEM medium, then underwent RNA extraction and qRT-PCR to analyze the gene expression. To investigate the in vivo physiological role of MTSS1, the male mice aged 8 
weeks were randomly allocated into 2 groups and received intra-tibial marrow transfection of either MTSS1 siRNA or control siRNA. The mice received the transfections twice in total, with an interval of 3 weeks. The mice were sacrificed 1 month after the 1 st transfection and the tibia samples were collected and subjected to histomorphological and immunohistochemical analyses.

\section{Histological staining and immunohistochemical staining}

After dissection, the tibiae samples were processed for sectioning and histological analyses. Briefly, the samples were fixed with $10 \%$ neutral buffered formalin for 3 days, decalcified with 14\% EDTA ( $\mathrm{pH} 7.4$ ) for 21 days, and embedded in paraffin. Sagittal sections were cut at $5 \mu \mathrm{m}$ thick.

For the overall observation of the bone and detection of adipocytes in the marrow, the sections were stained with hematoxylin and eosin (H\&E) following standard protocols. The quantity of the bone marrow fat was determined by measuring the numbers and areas of adipocytes on the sections. For the detection of osteoclasts, the sections were stained with tartrate-resistant acid phosphatase (TRAP) staining solution which consists of acetate buffer (0.1 M, pH 5.0), naphthol AS-MX phosphate $(0.1 \mathrm{mg} / \mathrm{ml})$, fast red violet LB salt $(0.6 \mathrm{mg} / \mathrm{mL})$, and sodium tartrate $(0.05 \mathrm{M})$. Osteoclast numbers and surfaces were determined. For the detection of osteoblasts, the sections were subjected to immunohistochemical staining. In brief, the paraffin-embedded samples were deparaffinized in xylene, and rehydrated in decreasing concentrations of ethanol, then digested with $0.05 \%$ trypsin at $37^{\circ} \mathrm{C}$ for $15 \mathrm{~min}$ for antigen retrieval. Endogenous peroxidases were quenched by incubation with $3 \%$ hydrogen peroxide for 5 minutes. Following blocking of nonspecific binding with $1 \% \mathrm{BSA}$, the sections 
were probed overnight at $4{ }^{\circ} \mathrm{C}$ with primary anti-ALP (Abcam) or anti-phospho-Src antibody (Affinity), then incubated with HRP-conjugated secondary antibody. The immunoreaction was developed with the chromogen substrate 3,3'-diaminobenzidine (DAB) and the nuclei were counterstained with hematoxylin. The numbers of ALP- and phospho-Src positive cells on the trabeculae were counted. The region of interest for the measurements starts from $0.1 \mathrm{~mm}$ below the growth plate and is $1 \mathrm{~mm}$ in length.

\section{Statistical analysis}

Data represent mean $\pm S D$. Student's t test and ANOVA test were conducted for comparisons between two groups and multiple groups, respectively. One-way ANOVA was applied when there is only one independent variable and two-way ANOVA was applied when there are two independent variables. Tukey test was conducted for post-hoc comparisons. A value of $p<0.05$ was regarded as significant.

Finding This work was funded by National Natural Science Foundation of China (81871741, 82072389, 82002264, 81972033 and 81972031).

Author contributions MC, LS, YG and LT performed the experiments. JZ, EZ and HY analyzed the data. XL supervised the research. BW designed the study, supervised the research, and wrote the manuscript. All authors read and approved the final manuscript.

Availability of data and material The data that support the findings of this study are available from the corresponding author upon reasonable request.

\section{Declarations}

Conflict of interest The authors declare that they have no competing interests.

Ethical approval The animal experiments were carried out following the National Standard of 


\section{Consent to participate Not applicable.}

408 Consent for publication Not applicable.

\section{References}

410 1. Chen Q, Shou P, Zheng C, Jiang M, Cao G, Yang Q, Cao J, Xie N, Velletri T, Zhang X, et al. (2016).

$411 \quad$ Fate decision of mesenchymal stem cells: adipocytes or osteoblasts? Cell Death Differ 23, 1128-1139.

412 2. Veldhuis-Vlug AG, and Rosen CJ (2017). Mechanisms of marrow adiposity and its implications for

3. Wu M, Chen G, and Li YP (2016). TGF-beta and BMP signaling in osteoblast, skeletal development,

4. Nakashima K, Zhou X, Kunkel G, Zhang Z, Deng JM, Behringer RR, and de Crombrugghe B (2002).

10. Xie F, Ye L, Ta M, Zhang L, and Jiang WG (2011). MTSS1: a multifunctional protein and its role in and bone formation. Cell 108, 17-29.

6. Salazar VS, Gamer LW, and Rosen V (2016). BMP signalling in skeletal development, disease and repair. Nat Rev Endocrinol 12, 203-221.

7. Lerner UH, and Ohlsson C (2015). The WNT system: background and its role in bone. J Intern Med $277,630-649$.

8. Mota de Sa P, Richard AJ, Hang H, and Stephens JM (2017). Transcriptional Regulation of Adipogenesis. Compr Physiol 7, 635-674.

9. Sadie-Van Gijsen H, Hough FS, and Ferris WF (2013). Determinants of bone marrow adiposity: the modulation of peroxisome proliferator-activated receptor-gamma2 activity as a central mechanism. Bone 56, 255-265.

11. Lee YG, Macoska JA, Korenchuk S, and Pienta KJ (2002). MIM, a potential metastasis suppressor cancer invasion and metastasis. Front Biosci (Schol Ed) 3, 621-631.

12. Du P, Wang S, Tang X, An C, Yang Y, and Jiang WG (2017). Reduced Expression of Metastasis Suppressor-1 (MTSS1) Accelerates Progression of Human Bladder Uroepithelium Cell Carcinoma. Anticancer Res 37, 4499-4505.

13. Du P, Ye L, Ruge F, Yang Y, and Jiang WG (2011). Metastasis suppressor-1, MTSS1, acts as a putative 
tumour suppressor in human bladder cancer. Anticancer Res 31, 3205-3212.

14. Wang Y, Liu J, Smith E, Zhou K, Liao J, Yang GY, Tan M, and Zhan X (2007). Downregulation of missing in metastasis gene (MIM) is associated with the progression of bladder transitional carcinomas. Cancer Invest 25, 79-86.

15. Yu J, Shen W, Gao B, Xu J, and Gong B (2020). Metastasis suppressor 1 acts as a tumor suppressor by inhibiting epithelial-to-mesenchymal transition in triple-negative breast cancer. Int J Biol Markers 35 , 74-81.

16. Zeleniak AE, Huang W, Fishel ML, and Hill R (2018). PTEN-Dependent Stabilization of MTSS1 Inhibits Metastatic Phenotype in Pancreatic Ductal Adenocarcinoma. Neoplasia 20, 12-24.

17. Chen L, Chen Q, Wu Y, Zhu M, Hu J, and Zhuang Z (2021). MTSS1 inhibits colorectal cancer metastasis by regulating the CXCR4/CXCL12 signaling axis. Int J Mol Med 47.

18. Wang H, Yu X, Wang X, Li X, and Yang S (2017). Missing in metastasis B, regulated by DNMT1, functions as a putative cancer suppressor in human lung giant-cell carcinoma. Acta Biochim Biophys Sin (Shanghai) 49, 238-245.

19. Mertz KD, Pathria G, Wagner C, Saarikangas J, Sboner A, Romanov J, Gschaider M, Lenz F, Neumann F, Schreiner W, et al. (2014). MTSS1 is a metastasis driver in a subset of human melanomas. Nat Commun 5, 3465 .

20. Huang XY, Huang ZL, Xu B, Chen Z, Re TJ, Zheng Q, and Tang ZY (2016). Elevated MTSS1 expression associated with metastasis and poor prognosis of residual hepatitis B-related hepatocellular carcinoma. J Exp Clin Cancer Res 35, 85.

21. Quinones GA, Jin J, and Oro AE (2010). I-BAR protein antagonism of endocytosis mediates directional sensing during guided cell migration. J Cell Biol 189, 353-367.

22. Bershteyn M, Atwood SX, Woo WM, Li M, and Oro AE (2010). MIM and cortactin antagonism regulates ciliogenesis and hedgehog signaling. Dev Cell 19, 270-283.

23. Brown AS, Meera P, Altindag B, Chopra R, Perkins EM, Paul S, Scoles DR, Tarapore E, Magri J, Huang H, et al. (2018). MTSS1/Src family kinase dysregulation underlies multiple inherited ataxias. Proc Natl Acad Sci U S A 115, E12407-E12416.

24. Soriano P, Montgomery C, Geske R, and Bradley A (1991). Targeted disruption of the c-src proto-oncogene leads to osteopetrosis in mice. Cell 64, 693-702.

25. Insogna KL, Sahni M, Grey AB, Tanaka S, Horne WC, Neff L, Mitnick M, Levy JB, and Baron R (1997). Colony-stimulating factor-1 induces cytoskeletal reorganization and c-src-dependent tyrosine phosphorylation of selected cellular proteins in rodent osteoclasts. J Clin Invest 100, 2476-2485.

26. Boyce BF, Yoneda T, Lowe C, Soriano P, and Mundy GR (1992). Requirement of pp60c-src expression for osteoclasts to form ruffled borders and resorb bone in mice. J Clin Invest 90, 1622-1627.

27. Marzia M, Sims NA, Voit S, Migliaccio S, Taranta A, Bernardini S, Faraggiana T, Yoneda T, Mundy GR, Boyce BF, et al. (2000). Decreased c-Src expression enhances osteoblast differentiation and bone formation. J Cell Biol 151, 311-320. 
28. Haraguchi K, Nishida A, Ishidate T, and Akiyama T (2004). Activation of beta-catenin-TCF-mediated transcription by non-receptor tyrosine kinase v-Src. Biochem Biophys Res Commun 313, 841-844.

29. Karni R, Gus Y, Dor Y, Meyuhas O, and Levitzki A (2005). Active Src elevates the expression of beta-catenin by enhancement of cap-dependent translation. Mol Cell Biol 25, 5031-5039.

30. Yokoyama N, and Malbon CC (2009). Dishevelled-2 docks and activates Src in a Wnt-dependent manner. J Cell Sci 122, 4439-4451.

31. Zhang QQ, Zhou DL, Lei Y, Zheng L, Chen SX, Gou HJ, Gu QL, He XD, Lan T, Qi CL, et al. (2015). Slit2/Robo1 signaling promotes intestinal tumorigenesis through Src-mediated activation of the Wnt/beta-catenin pathway. Oncotarget 6, 3123-3135.

32. Condello S, Cao L, and Matei D (2013). Tissue transglutaminase regulates beta-catenin signaling through a c-Src-dependent mechanism. FASEB J 27, 3100-3112.

33. Chen Q, Su Y, Wesslowski J, Hagemann AI, Ramialison M, Wittbrodt J, Scholpp S, and Davidson G (2014). Tyrosine phosphorylation of LRP6 by Src and Fer inhibits Wnt/beta-catenin signalling. EMBO Rep 15, 1254-1267. 
490

491

492

493

494

495

496

497

498

499

500

501

502

\section{Figure legends}

\section{Figure 1. MTSS1 was upregulated during osteoblast differentiation and downregulated}

in ovariectomized and aged mice. MTSS1 mRNA expression was examined in various tissues of mice using qRT-PCR. The level of MTSS1 in colon was set at 1 (A). MTSS1 expression was examined using qRT-PCR in ST2 cells at indicated time points during osteoblast differentiation (B). MTSS1 expression was examined in the BMSCs of ovariectomized mice (C) or in the calvaria of 18-month-old mice (D). Values represent mean \pm SD, $n=3$. One-way ANOVA was conducted in (B) followed by Tukey test for post-hoc comparisons. Student's t test was conducted in (C, D). *Significant vs. day $0(B)$, sham (C) or 3-month-old mice (D), $p<0.05$.

Figure 2. Silencing of MTSS1 inhibited osteogenic differentiation of stromal progenitor cells. The knockdown of MTSS1 was verified using qRT-PCR in primary BMSCs isolated from 6-week-old mice (A) and ST2 cells (E) after infection of MTSS1 shRNA expression lentivirus or transfection of MTSS1 siRNAs. BMSCs or ST2 cells underexpressing MTSS1 were induced to allow osteogenic differentiation. The effects of MTSS1 silencing (BMSCs: B-D; ST2: F-H) on osteoblast differentiation were examined. alizarin red staining and/or ALP staining were performed in differentiated osteoblasts 14 days and 21 days, respectively, after osteogenic treatment $(B, F)$. The mRNA $(C, G)$ and protein $(D, H)$ levels of osteogenic factors were measured $72 \mathrm{~h}$ after osteogenic treatment. Values represent mean $\pm S D, n=3$. Student's $t$ test was conducted in (A, C, D). One-way ANOVA was conducted in (E, G, H) followed by Tukey test for post-hoc comparisons. *Significant vs. control LV or control siRNA, $\mathrm{p}<0.05$.

Figure 3. Overexpression of MTSS1 stimulated osteogenic differentiation of stromal 
progenitor cells. The overexpression of MTSS1 was verified using qRT-PCR in ST2 cells after transfection of MTSS1 expression construct (A). The ST2 cells after transfection were

514 induced to allow osteogenic differentiation. The effect of MTSS1 overexpression on osteoblast differentiation was examined. ALP staining was performed in differentiated osteoblasts 14 days after osteogenic treatment (B). The mRNA (C) and protein (D) levels of osteogenic factors were measured $72 \mathrm{~h}$ after osteogenic treatment. The effect of MTSS1 overexpression on cell growth rate of ST2 cells was measured using a CCK-8 kit (E). Values represent mean $\pm S D, n=3$. Student's t test was conducted. *Significant vs. vector, $p<0.05$.

Figure 4. MTSS1 inactivated Src and activated Wnt/ß-catenin signaling in stromal

progenitor cells. ST2 cells were transfected with MTSS1 expression construct (A, D ) or

MTSS1 siRNA (B, E) or Src expression construct $(F)$ and their control. The protein levels of MTSS1, phosphorylated and total Src were detected in the cells 3 days after transfection using Western blotting (A, B). Co-IP was performed and endogenous MTSS1 and LRP6 were detected with Western blotting (C). The protein levels of the major components of Wnt/ $\beta$-catenin signaling were detected in the cells 3 days after transfection using Western blotting $(D-F)$. Values represent mean $\pm S D, n=3$. Student's $t$ test was conducted in $(A, D, F)$. One-way ANOVA was conducted in (B, E) followed by Tukey test for post-hoc comparisons. *Significant vs. vector or control siRNA, $\mathrm{p}<0.05$

Figure 5. Overexpression of Src impaired osteogenic differentiation of progenitor cells.

The overexpression of Src was verified using qRT-PCR in ST2 cells after transfection of Src expression construct (A). The ST2 cells after transfection were induced to allow osteogenic differentiation. The effect of Src overexpression on osteoblast differentiation was examined. 
ALP staining was performed in differentiated osteoblasts 14 days after osteogenic treatment

(B). The mRNA (C) and protein (D) levels of osteogenic factors were measured $72 \mathrm{~h}$ after osteogenic treatment. Src mRNA level was examined using qRT-PCR in the BMSCs of 16 week-old mice 8 weeks after ovariectomy (Fig. $5 E$ ). Values represent mean $\pm S D, n=3$. Student's t test was conducted. *Significant vs. vector, $\mathrm{p}<0.05$.

Figure 6. Src relieved the stimulation of osteogenic differentiation induced by MTSS1.

ST2 cells were cotransfected with MTSS1 and Src expression construct or the vector, and were induced to allow osteogenic differentiation (A-C). Alongside, ST2 cells were transfected with control or MTSS1 siRNA, then induced to allow osteogenic differentiation in the osteogenic medium supplemented with $10 \mu \mathrm{m}$ Src inhibitor 1 (Src-I1) (D-F). ALP staining was performed in differentiated osteoblasts 14 days after osteogenic treatment $(A, D)$. The mRNA $(B, E)$ and protein $(C, F)$ levels of osteogenic factors were measured $72 \mathrm{~h}$ after osteogenic treatment. Values represent mean $\pm S D, n=3$. Two-way ANOVA was conducted in followed by

Tukey test for post-hoc comparisons. *Significant vs. Vector or control siRNA+DMSO, "Significant vs. Vector+MTSS1 or MTSS1 siRNA+DMSO, $\mathrm{p}<0.05$.

Figure 7. Silencing of MTSS1 reduced trabecular bone mass in mice. qRT-PCR $(A)$ and

Western blotting (B) were done to verify the silencing of MTSS1 mRNA in the BMSCs isolated $48 \mathrm{~h}$ after in vivo transfection of MTSS1 siRNA. Three-dimensional reconstruction of $\mu \mathrm{CT}$ images are shown $(C)$ and histomorphometric parameters were measured (D-G) in the mice was conducted. * $\mathrm{p}<0.05$ vs. Control siRNA.

Figure 8. Silencing of MTSS1 impaired the differentiation of osteoblasts in mice. 
556 Histological, histomorphometric and immunohistochemical analyses were performed on the

557 bone sections from the mice 4 weeks after transfection. H\&E staining was done. Image scale:

$558500 \mu \mathrm{m}(\mathrm{A})$. Numbers (B) and areas (C) of adipocytes were quantified. Representative 559 images of immunohistochemical staining for ALP (D) and phospho-Src (F) are shown. Image 560 scale: $20 \mu \mathrm{m}(\mathrm{D}, \mathrm{F})$. Numbers of ALP-positive osteoblasts (E) and phospho-Src-positive cells 561 on the trabeculae were counted (G). Representative images of TRAP staining are shown. 562 Image scale: $20 \mu \mathrm{m}(\mathrm{H})$. Numbers of osteoclasts were counted (I). The mRNA expression of 563 RANKL and OPG was detected in ST2 cells following MTSS1 overexpression and the ratio 564 was calculated $(\mathrm{J})$. Data are mean $\pm \mathrm{SD}$. $\mathrm{n}=6$ in $(\mathrm{B}, \mathrm{C}, \mathrm{E}, \mathrm{G}, \mathrm{I}, \mathrm{J})$. Student's $\mathrm{t}$ test was conducted. ${ }^{*} \mathrm{p}<0.05$ vs. Control siRNA. $\mathrm{n}=3$ in $(\mathrm{J}),{ }^{*} \mathrm{p}<0.05$ vs. Vector.

566 Figure 9. Schematic diagram depicting the mechanism for MTSS1 in regulating osteoblast 567 differentiation. 
Relative MTSS1 mRNA D (Fold change)

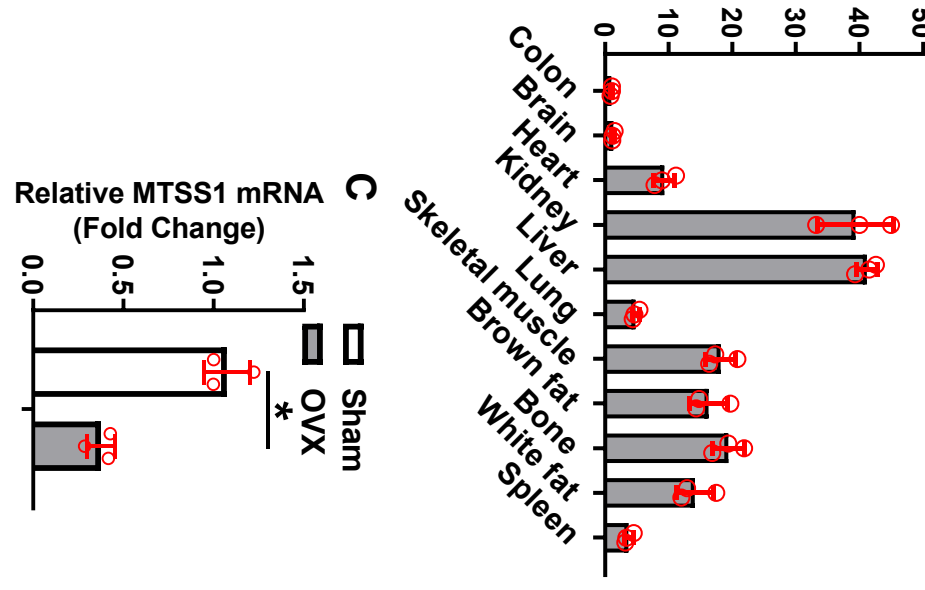

Relative MTSS1 mRNA $\square$ (Fold Change)

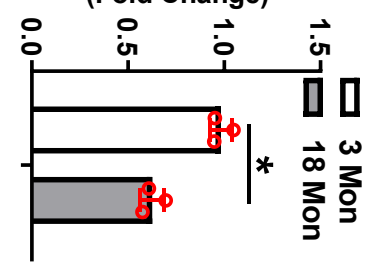

Relative MTSS1 mRNA

(Fold change)

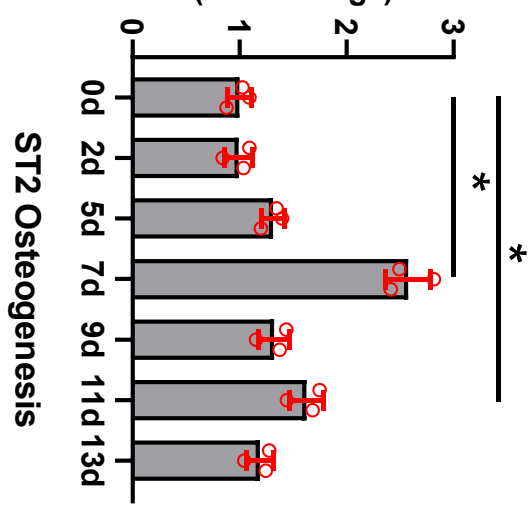


Figure 2
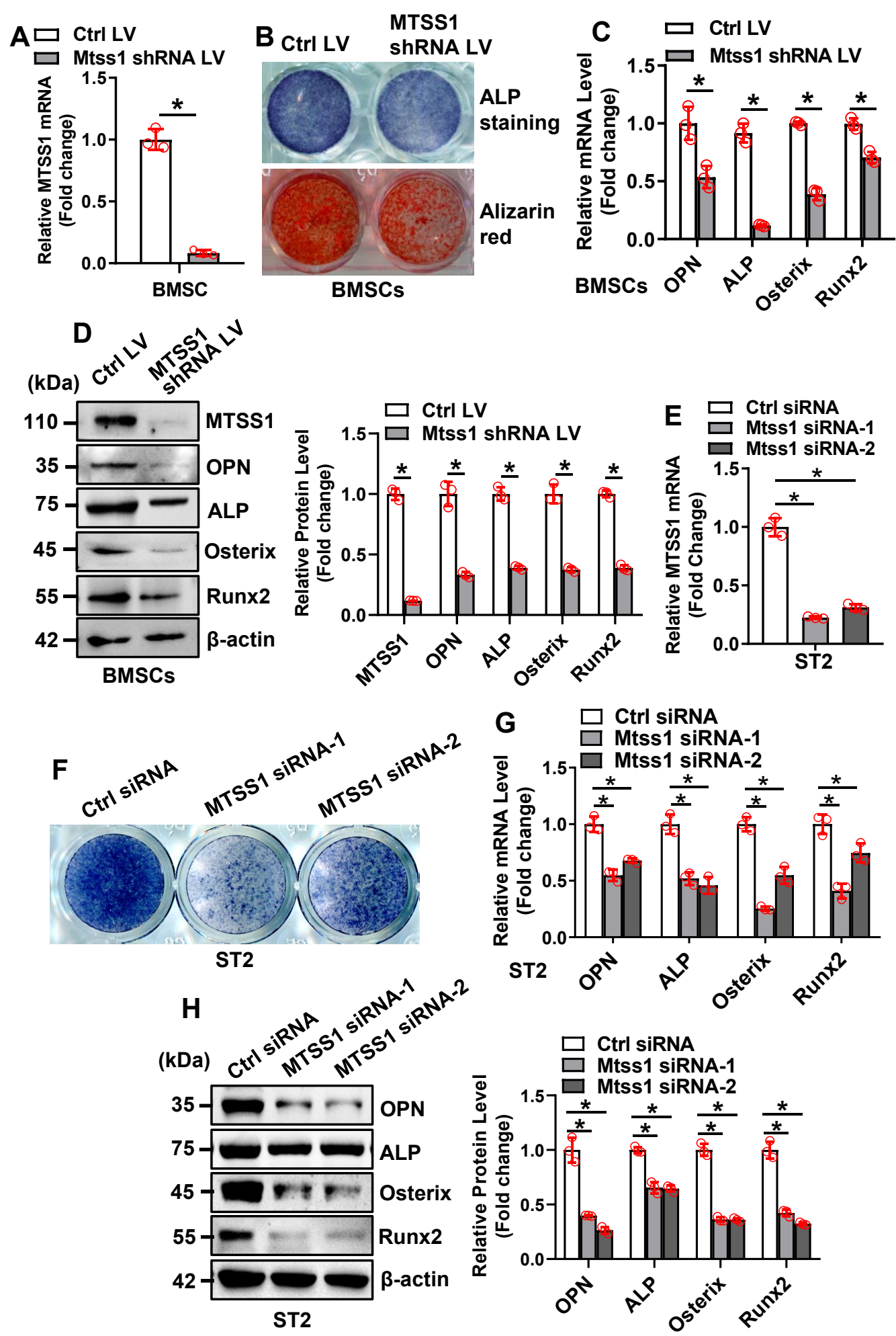
Figure 3
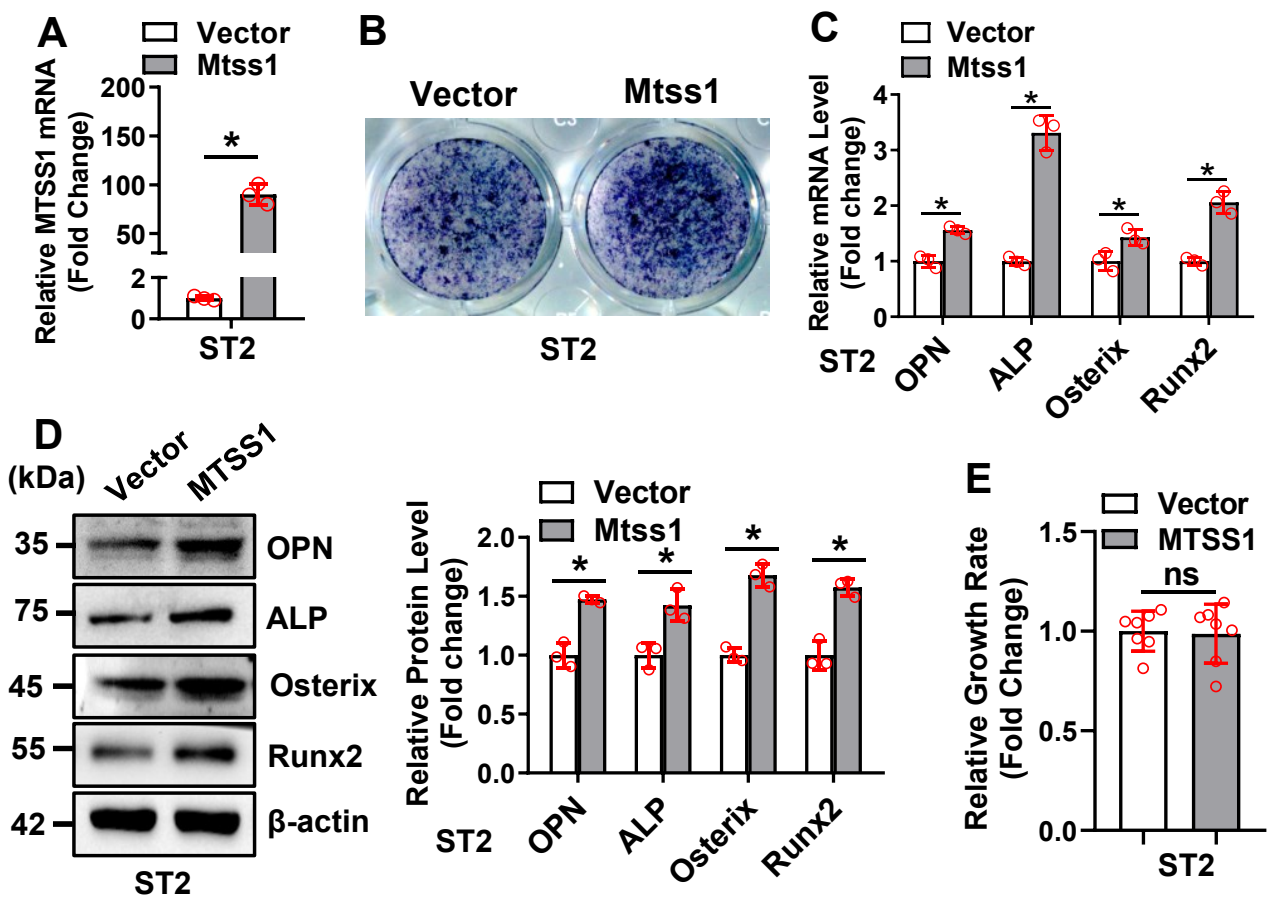
Figure 4

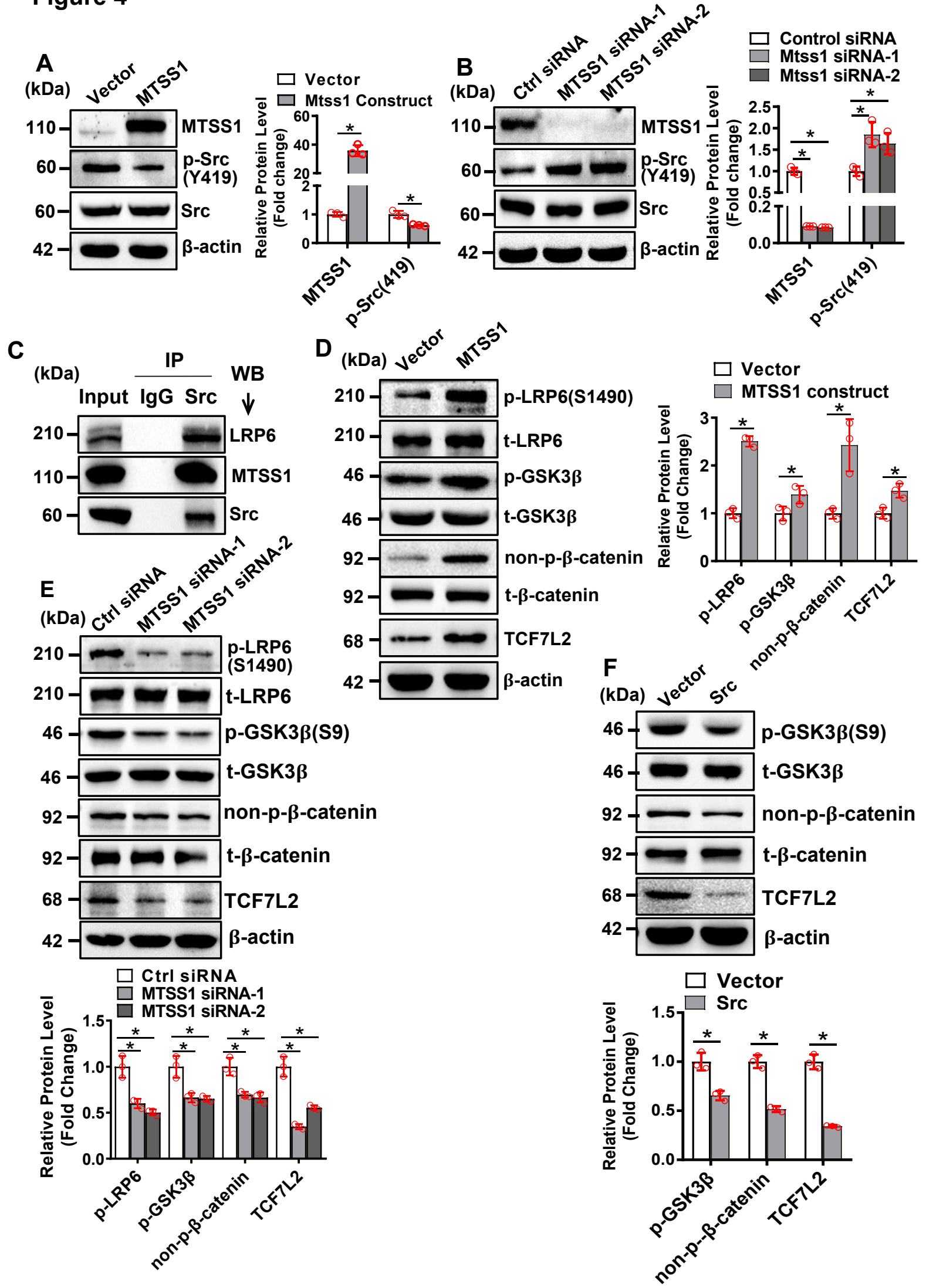


Figure 5
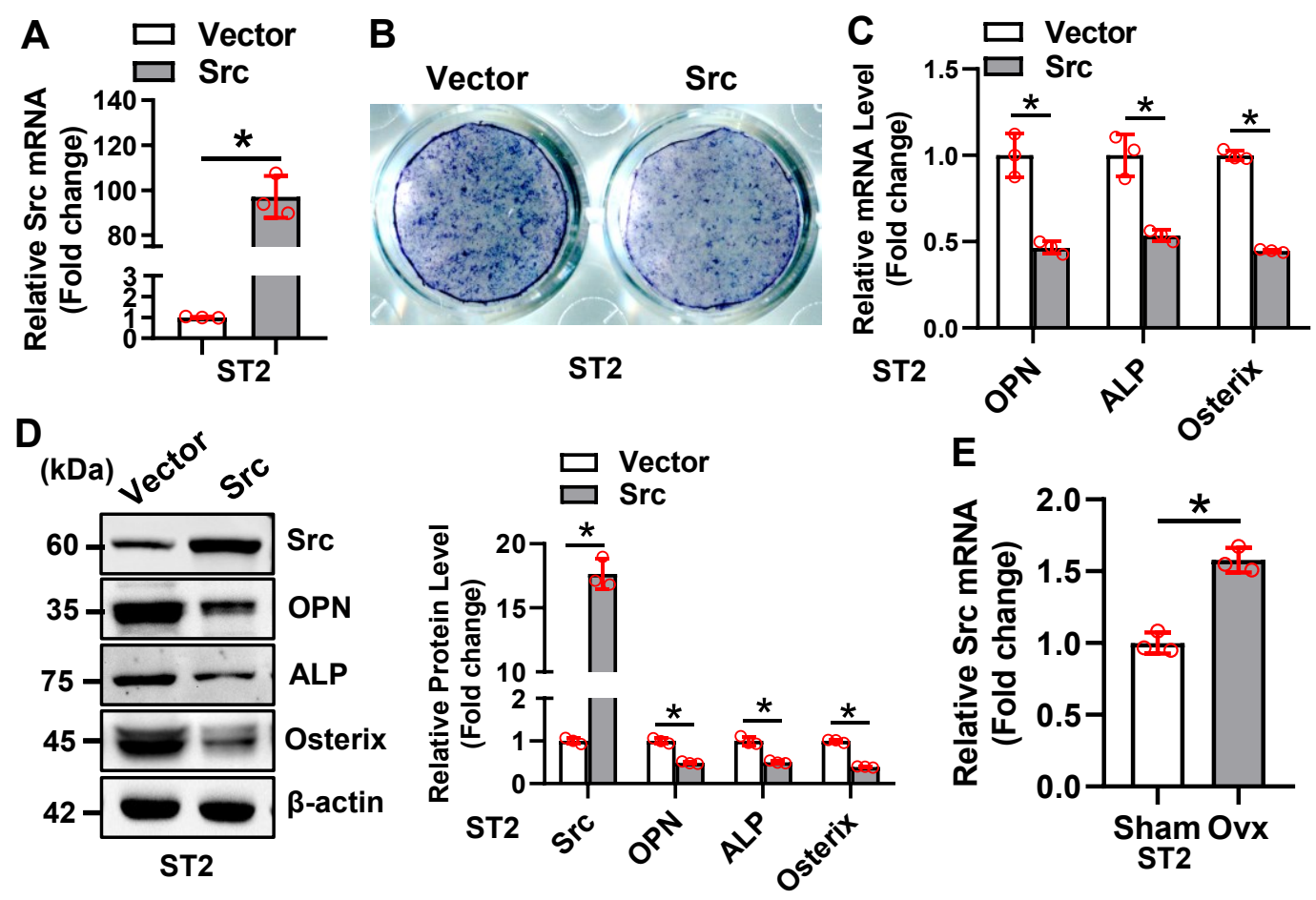
Figure 6

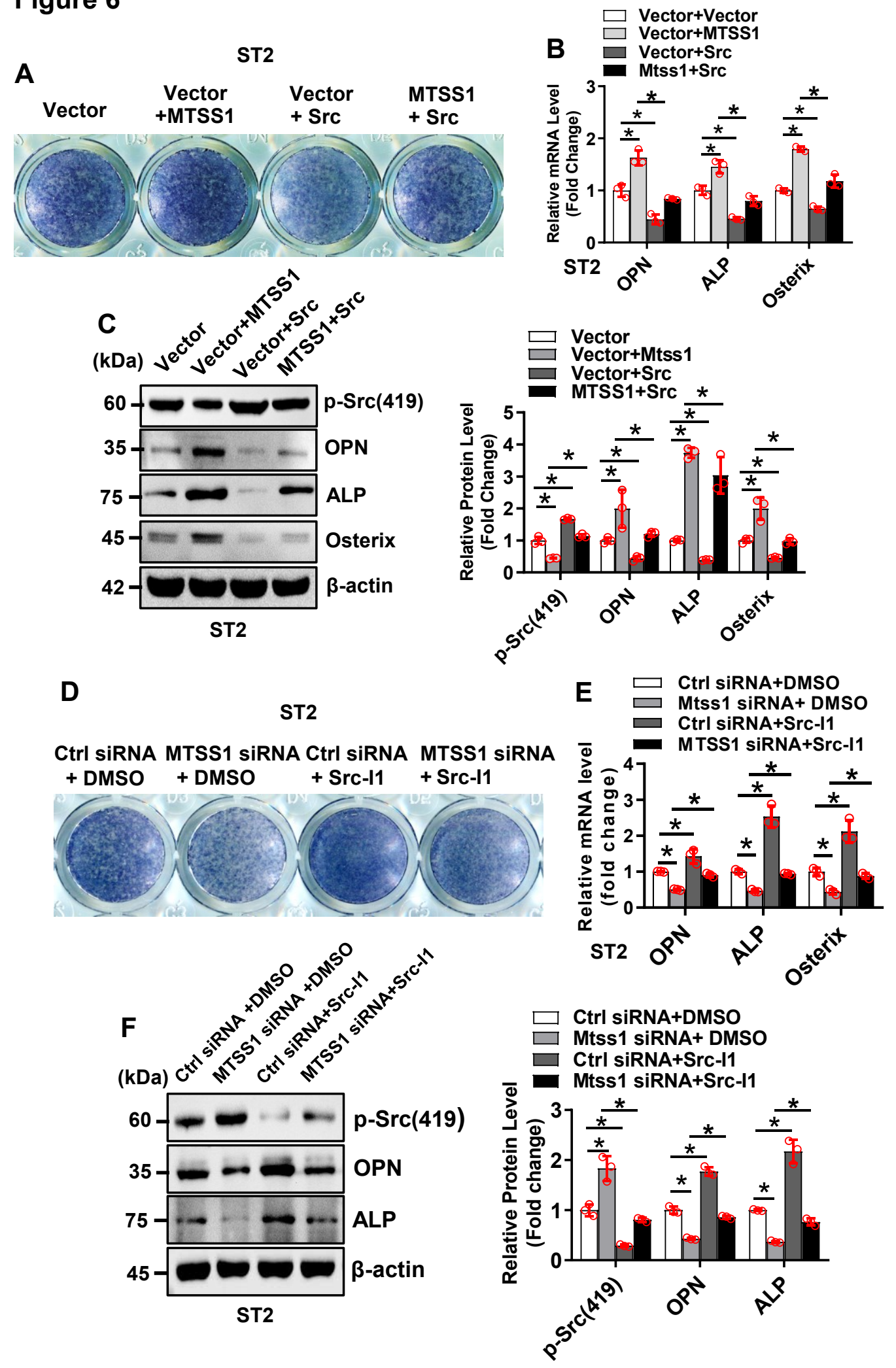


Figure 7

A Ctrl siRnA
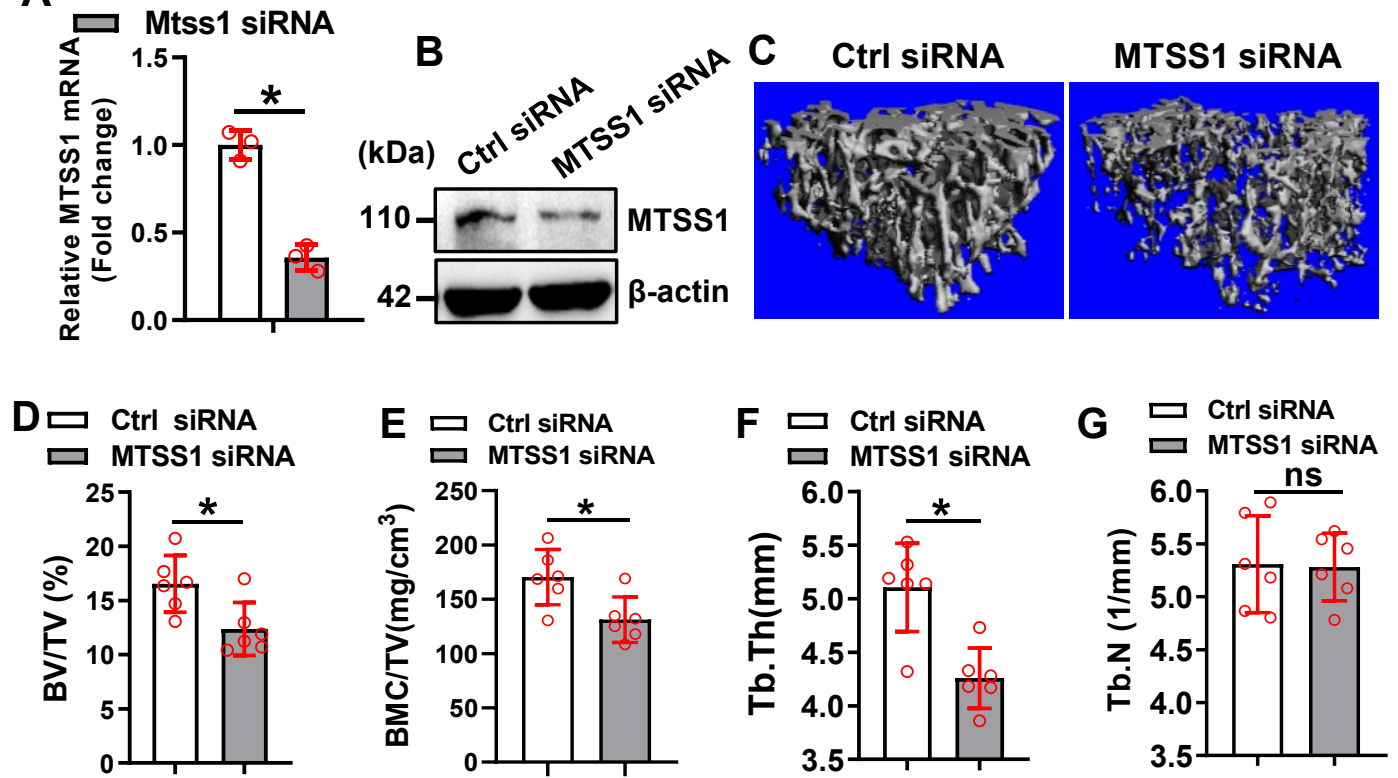
Figure 8

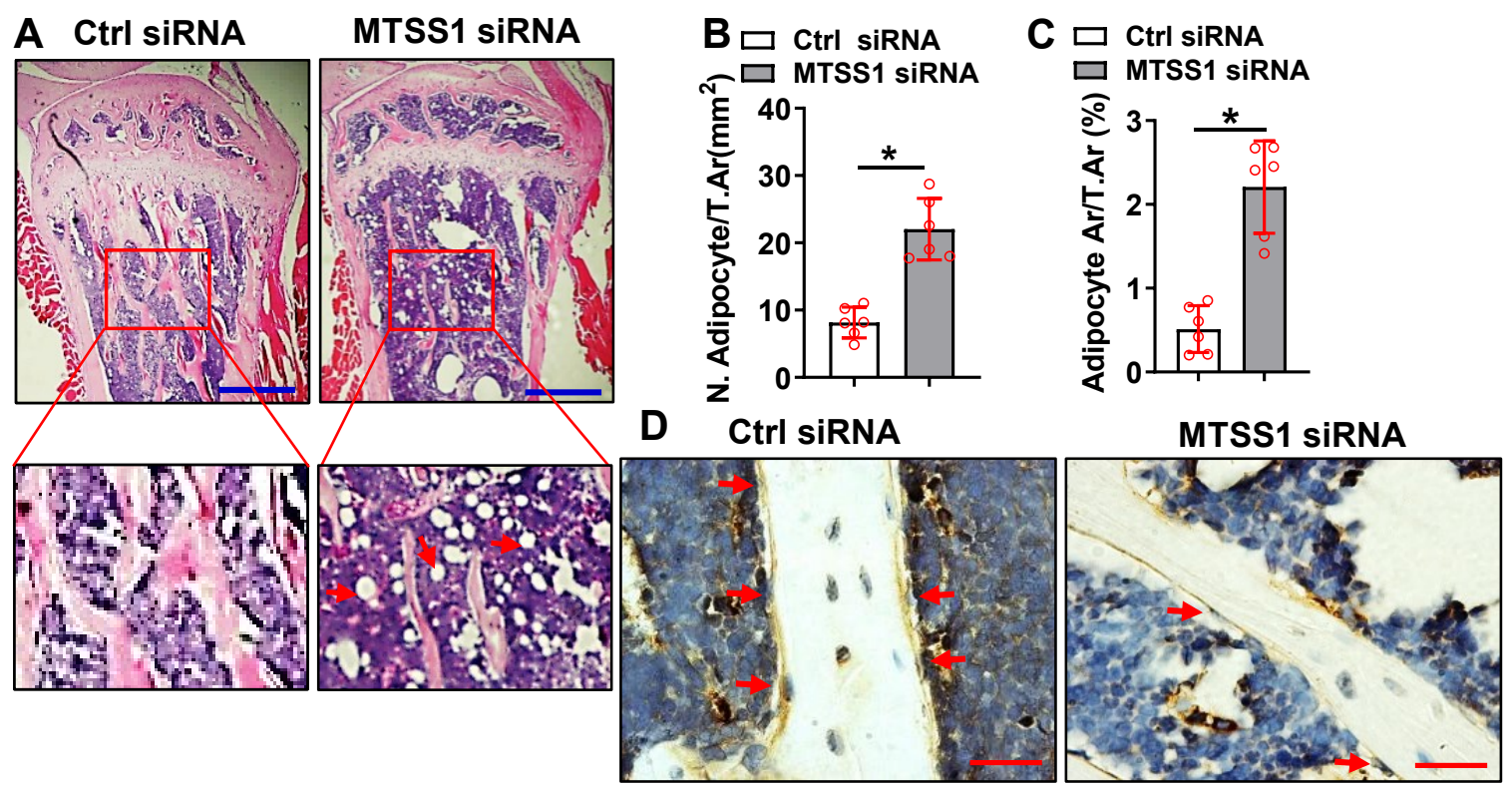

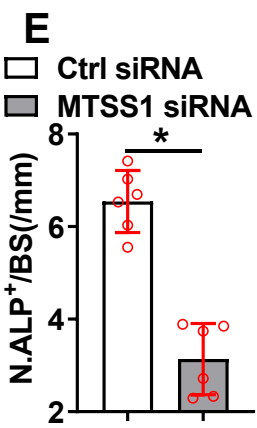
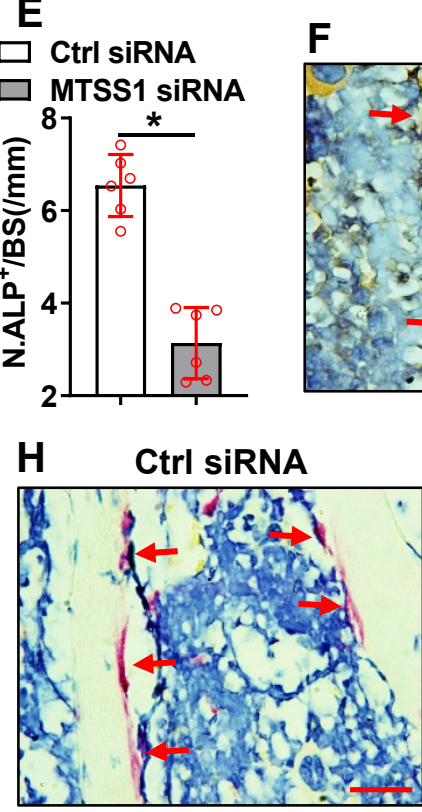
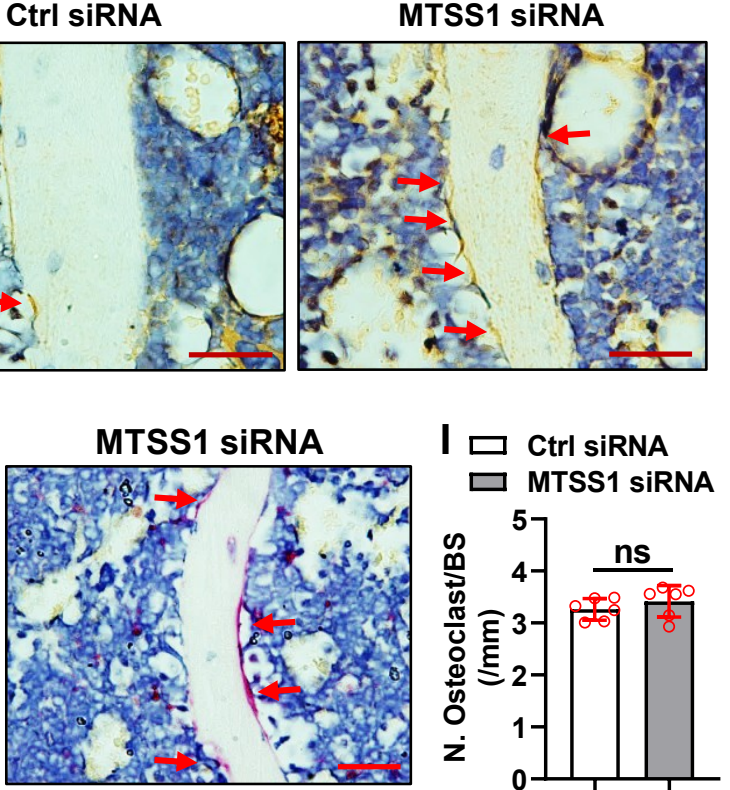

G
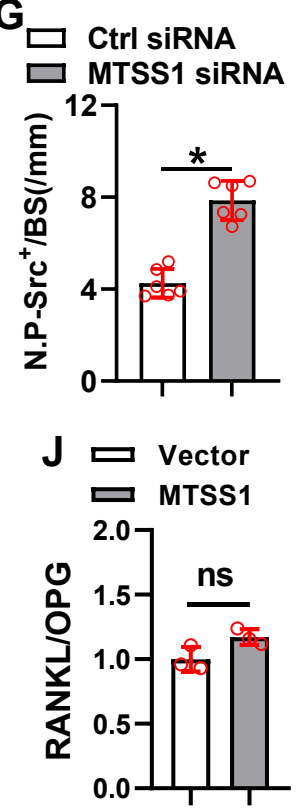
Figure 9

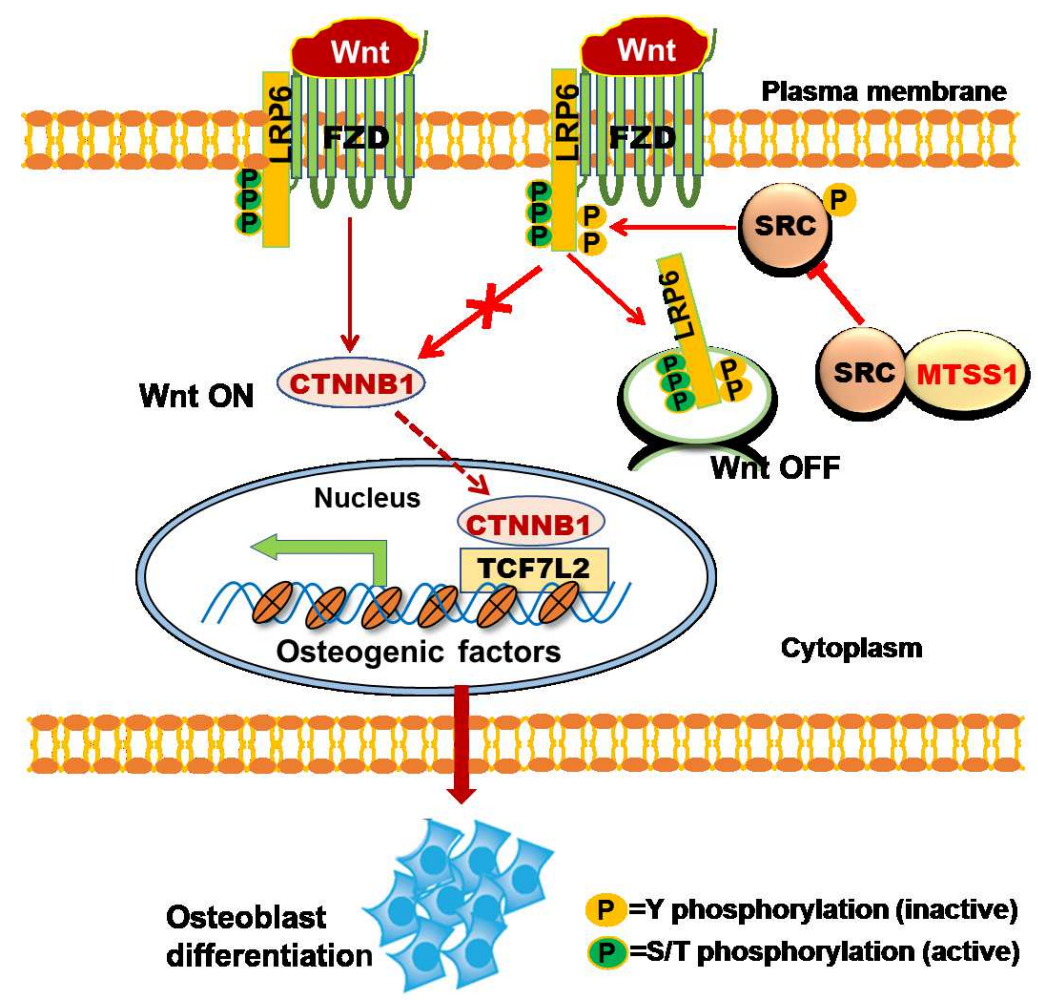


Table S1. Primers used for qRT-PCR and full-length cDNA amplification

\begin{tabular}{|c|c|c|}
\hline Genes & Forward primer sequences: & Reverse primer sequences \\
\hline Runx2 & TCCTGTAGATCCGAGCACCA & CTGCTGCTGTTGTTGCTGTT \\
\hline Alp & CCAGAAAGACACCTTGACTGTGG & TCTTGTCCGTGTCGCTCACCAT \\
\hline Osterix & GGCTTTTCTGCGGCAAGAGGTT & CGCTGATGTTTGCTCAAGTGGTC \\
\hline Bsp & AATGGAGACGGCGATAGTTCCG & GGAAAGTGTGGAGTTCTCTGCC \\
\hline Opn & GCTTGGCTTATGGACTGAGGTC & CCTTAGACTCACCGCTCTTCATG \\
\hline$\beta$-actin & AAGACCTCTATGCCAACACAG & GGAGGAGCAATGATCTTGATC \\
\hline Mtss1 & CACCTGAAGCTGCCAACCAGTT & AGCGTAGTCAGGAAGGTGGACA \\
\hline Src & GTTGCTTCGGAGAGGTGTGGAT & CACCAGTTTCTCGTGCCTCAGT \\
\hline $\begin{array}{l}\text { Src full-length } \\
\text { cloning }\end{array}$ & $\begin{array}{l}\text { TTGGTACCGAGCTCGGATCCGCCACC } \\
\text { ATGGGCAGCAACAAGAGCAA }\end{array}$ & $\begin{array}{l}\text { GCTGGATATCTGCAGAATTCCTAT } \\
\text { AGGTTCTCCCCGGGCTGGTACT }\end{array}$ \\
\hline $\begin{array}{l}\text { Mtss1 shRNA } \\
\text { oligo pair }\end{array}$ & $\begin{array}{l}\text { GATCCGTAGACAACTGGTTGGCAGC } \\
\text { TTCAAGAGAGCTGCCAACCAGTTGT } \\
\text { CTATTTTTTACGCGTG }\end{array}$ & $\begin{array}{l}\text { AATTCACGCGTAAAAAATAGACAAC } \\
\text { TGGTTGGCAGCTCTCTTGAAGCTG } \\
\text { CCAACCAGTTGTCTACG }\end{array}$ \\
\hline
\end{tabular}


Table S2. siRNA sequences used for gene silencing

\begin{tabular}{lll}
\hline Genes & Sense sequences & Antisense sequences \\
\hline Mtss1 siRNA-1 & GCAAUAUACCCUCUUCCUUTT & AAGGAAGAGGGUAUAUUGCTT \\
Mtss1 siRNA-2 & GCUGCCAACCAGUUGUCUATT & UAGACAACUGGUUGGCAGCTT \\
\hline
\end{tabular}

